\title{
Occurrence Patterns, Photoperiod and Dispersion Ability of the Non-Marine Ostracoda (Crustacea) in Shallow Waters
}

\author{
Okan Külköylüoğlu ${ }^{1, *} \mathbb{B}$, Mehmet Yavuzatmaca ${ }^{1}$, Derya Akdemir $^{2}$
}

\begin{abstract}
${ }^{1}$ Bolu Abant İzzet Baysal University, Faculty of Arts and Science, Department of Biology, Bolu 14280 Turkey. ${ }^{2}$ Cologne University, Institute of Geology and Mineralogy, Faculty of Mathematics and Natural Sciences, 50935, Köln, Germany.
\end{abstract}

\section{Article History}

Received 14 July 2020

Accepted 01 December 2020

First Online 02 December 2020

\section{Corresponding Author}

Tel.: +903742541000

E-mail: kulkoyluoglu_o@ibu.edu.tr

\section{Keywords}

Cosmoecious species

Habitat changes

Ecological tolerance

Afterward-cosmopolitan

\begin{abstract}
Patterns of species occurrence, dispersion ability, habitat preferences and sampling time can be important factors on the species composition. To understand effective roles of these factors on non-marine ostracods, samples were collected from 98 different shallow aquatic bodies from Osmaniye and Kilis provinces in Turkey. Total of 16 and 12 species were identified from the two provinces, respectively. All species are reported new for these provinces. Three species (Heterocypris incongruens, llyocypris inermis, I. bradyi) demonstrated the most frequent occurrences and abundances in up to seven different habitats. Species diversity and abundance were at least two times higher in natural habitats (streams, creeks) than artificial habitats (reservoirs, troughs). Numbers of species with and without swimming setae on the second antenna was not significantly different between lentic and lotic habitats. A positive co-occurrence pattern was found between Neglecandona neglecta and $I$. inermis while the rest of the species pairs exhibited random co-occurrences to each other. Canonical Correspondence Analysis showed $80.8 \%$ of correlation between species and environmental variables when water temperature was the major effective factor $(\mathrm{P}<0.05)$ on species occurrence. Sampling time did not make difference on the numbers of species between morning (06:30-11:58 a.m.) and after noon (12:05-19:52 p.m.). Results suggest that species occurrence seems to be related to species-specific characteristics in its $n$-dimensional niche where species deals with several other factors.
\end{abstract}

\section{Introduction}

Ostracods are small aquatic crustaceans that can be found from variety of aquatic habitats, presenting species-specific habitat preferences (Külköylüoğlu and Vinyard, 2000). They are known as one of the largest taxonomic groups observed from $5000 \mathrm{~m}$ below (Benson, 1972; Brandão and Yasuhara, 2013) to $4000 \mathrm{~m}$ above (Laprida, et al., 2006; Mischke et al., 2007) sea level. Indeed, Fürstenberg et al. (2015) reported live individuals of Leucocytherella sinensis from Lake Puma Yumco located at about $5031 \mathrm{~m}$ a.s.l. in Tibetian Plateau. Such a wide range $(>9000 \mathrm{~m}$ ) of dispersing ability may correspond to their high levels of tolerances to different environmental factors, higher adaptive values, desiccation resistant eggs, relatively high reproductive rates, and small size. Moreover, individual species may prefer specific conditions in certain type of habitats. For example, Külköylüoğlu et al. (2003) reported that a hot spring ostracod species Thermopsis thermophila was only found in hot springs of western states in the United States of America ranging from $36^{\circ} \mathrm{C}$ to $55^{\circ} \mathrm{C}$. This endemic species was found all year round in those thermal spring habitats within this range and does not survive below $36^{\circ} \mathrm{C}$. Besides, occurrence frequencies and abundance of the species correspond with 
decreasing water temperature. Moreover, species occurrence (presence or absence data) can be associated to several factors such as habitat types and conditions, species dispersion ability (active or passive dispersion), and sampling time (before and after noon).

Processes in global perspective such as climatic change and anthropogenic factors are generally considered as two of the most important drivers of changes in heterogeneity of aquatic ecosystems (Rahel and Olden, 2008; Belmecheri et al., 2009; Hart and Gotelli, 2011). Accordingly, aquatic habitat type influenced by these kind of large-scaled factors can be temporarily or permanently transformed to another type in a relatively short period of time, influencing species community and diversity of that of habitats. As a result, these processes can be effective on the function, structure and dynamics of species populations, communities and ecosystems (Panuccio et al., 2019). For example, artificial modification of the natural spring habitats to troughs (Külköylüoğlu, 2003) was shown to cause critical changes in ostracod species assemblages where numbers of cosmopolitan species (i.e., species with wide geographical distributions) were eventually increased (Uçak et al., 2014; Külköylüoğlu et al., 2016).

This is an important issue from the conservation and ecological aspect where correct application of data on aquatic invertebrates is commonly used for biomonitoring studies with different biotic indices and/or metrics. For example, Gregory (2005) showed that different microhabitat preferences of aquatic invertebrates in 10 different streams in Georgia and Alabama influenced the results of such bio-assessment applications. Overall, these above studies indicated that microhabitat structure exerts important influence on aquatic invertebrate (e.g., ostracods) assemblages due to the changes in habitat structure and function. In such a case, species may respond differently to habitat changes. This may cause changes inpopulation structure in that of particular habitat(s) causing a possible shift in some ostracod species from native/rare to cosmopolitans. In the meantime, habitat changes might be favorable for some species to increase their abundance values and eventually extend their geographical distribution. This means that once a species is rare or endemic in a particular habitat type it may have gain cosmopolitan characteristics after conditions are changed favoring for its survival (Külköylüoğlu et al., 2003). These type of species (once rare but later can have a cosmopolitan distribution) may be called "afterward-cosmopolitan" species (Külköylüoğlu, this study). This theoretical term used in here does not imply invasive species which are actually not the parts of that of natural habitats but are introduced -deliberately and/or inadvertently- into a new region (Ricciardi, 2013) or transferred in to the areas where species can increase its survival chances. It is therefore imperative to understand the future possibilities of habitat changes under the pressure of effective factors. Moreover, habitat changes along with the chances in conditions can also affect species cooccurrence patterns. Species with positive cooccurrences and with similar habitat preferences will show more similar reactions to such changes in habitats than species with negative co-occurrences. Therefore, studying the effect of habitat changes at species level (i.e., ostracods) or even higher taxonomic levels can help us to understand future possibilities of species composition and diversity (Külköylüoğlu et al., 2012a). Such perspective requires two basic understandings on (1) present species composition, and (2) their habitat preferences. Once these two are known, it can be possible to make future estimates for changes in particular habitats. For example, survival chance of individual species can be predicted when habitat is changed to another type (Uçak et al., 2014).

Ostracod occurrences can be related to their active or passive distribution modes among water bodies. In active distribution, they can swim or walk within short distances. In passive distribution, either individual species or their sticky eggs can be carried out by hosts such as wind (Moreno et al., 2016), flowing water (Havel et al., 2000), fish (Vinyard, 1979), aquatic birds (Proctor et al., 1967), frogs and snakes (Lopez et al., 1999), mammals (Wanschoenwinkel et al., 2008) and humans (Valls et al., 2017).

Akdemir et al. (2016) showed that ostracod species without swimming ability may have different habitat preferences among seven different aquatic habitats in Gaziantep, Turkey. The authors underlined the fact that these species appeared to prefer lotic habitats more than lentic. Külköylüoğlu et al. (2017a) and Külköylüoğlu et al. (2017b) suggest that some ostracods do have tendencies for certain kinds of aquatic conditions in specific habitats such as wetlands. Possible outline of these studies implied that sampling time (e.g., daily, monthly or seasonal samplings) may also be considered as influential factor on species occurrences. Accordingly, during the present study, we examine the best sampling period at the daylight in order to collect ostracods in activity and abundance. This approach is based on the study of Ganning (1967) who observed that the presence of ostracod species Heterocypris salina at higher abundances and occurrences in early morning compared to after noon was due to the species' negative response to light. Ganning's observations deserve further interest into the factors related to ostracods occurrence probabilities which consist of both abundance and richness values. Accordingly, species with similar ecological (and/or behavioral) characteristics and responds can have similar patterns. Therefore, sampling time of a day may play critical role on their abundances and occurrences in variety of habitats. As far as we know, Ganning's observations have not been tested in field conditions yet. Thereby, we wanted to be the first to test his observations in our field work. Thus, the aims of this study are i) to document non-marine ostracod diversity/composition in this region of southern Turkey for the first time, ii) to 
compare occurrence patterns of swimmer and/or nonswimmer species, iii) to estimate species tolerance and optimum values for particular environmental variables, and iv) to test the hypothesis that there is no difference in the mean numbers of species and occurrences before and after noon samplings.

\section{Materials and Methods}

\section{Site Description}

Osmaniye $\left(3,279.9 \mathrm{~km}^{2}\right)$ and Kilis $\left(1,642 \mathrm{~km}^{2}\right)$ are two provinces located in the southern part of Turkey. Kilis is situated near the border with Syria and about 80 $\mathrm{km}$ east of the Mediterranean Sea. Osmaniye is located $23 \mathrm{~km}$ northwest of Kilis with about $30 \mathrm{~km}$ distance to the sea (Figure 1). There are basically three reasons for choosing these provinces: i) non-marine ostracod distributions are not well known in both provinces; therefore, all findings are new and unique for the provinces, ii) there is no geographical connection between the provinces where another province (Gaziantep) stretches its border between them. Ostracod species composition of Gaziantep is already known (Akdemir et al., 2016). Additionally, geographical regions that these provinces belong to are known to have different climatic conditions. While Osmaniye is in the Mediterranean Region, Kilis is located end of Southeast Anatolian region. According to KöppenGeiger climate classification, both regions fit into Warm temperate climate zone with dry and hot summers (Kottek et al., 2006) where the mean air temperature and rainfall is about $17^{\circ} \mathrm{C}$ and $505 \mathrm{~mm}$ a year in Kilis and $18.5^{\circ} \mathrm{C}$ and $855 \mathrm{~mm}$ in Osmaniye (TSMS, 2020). Accordingly, we assume that ostracod composition can also be sufficiently compared among the provinces. This is also because all the provinces compared in the study bear similar aquatic types (although the diversity of these habitat types may be different), and iii) both provinces have a variety of aquatic bodies at different elevational ranges. This is important because possible correlations between species occurrence and elevation have been critically argued in the literature (see e.g., Yavuzatmaca et al., 2018, and references in there). Therefore, we consider that our study may provide sufficient evidence of the importance of elevation on species occurrences.

\section{Sampling and Measurements}

We visited 98 different sites with 10 different habitat types (e.g., spring, creek, ditch, pool, reservoir, river, stream, trough, wetland and canal) between 06:30 a.m. and 19:52 p.m. further-eastern European time during 14-17 May, 2015. In total, 13 environmental variables $\left(\mathrm{pH}\right.$; dissolved oxygen $\left(\mathrm{mg} \mathrm{l}^{-1}\right)$; percent oxygen saturation (\% sat.); water temperature (으); air temperature $(\stackrel{\circ}{ } \mathrm{C})$; electrical conductivity $\left(\mu \mathrm{S} \mathrm{cm}^{-1}\right)$; total dissolved solids ( $\mathrm{mg} \mathrm{l}^{-1}$ ); salinity (ppt); atmospheric pressure $(\mathrm{mmHg})$; Standard Hydrogen Electrode $(\mathrm{mV})$; air moisture (\%); wind speed $\left(\mathrm{m} \mathrm{s}^{-1}\right)$; elevation $(\mathrm{m})$ ) were measured in situ. The anemometer (Testo 410-2) was used to measure air temperature, wind speed, and air moisture while geographical data (elevation, coordinates) were recorded with a geographical positioning system (GARMIN etrex Vista H GPS). All other measurements were taken with a YSI-Multiprobe Professional Plus. Sediment and water samples were randomly collected from each site (ca. 5-100 cm water depth) by means of using hand nets ( $200 \mu \mathrm{m}$ mesh size) after measurements were taken (see details in Yavuzatmaca et al., 2017). Each sample was fixed with $70 \%$ ethanol in $250 \mathrm{ml}$ plastic bottles and transferred to the laboratory where samples were filtered through four standardized sieves $(0.5,1.0,1.5$ and $2.0 \mathrm{~mm}$ mesh size) under tap water and kept in $70 \%$ ethanol for

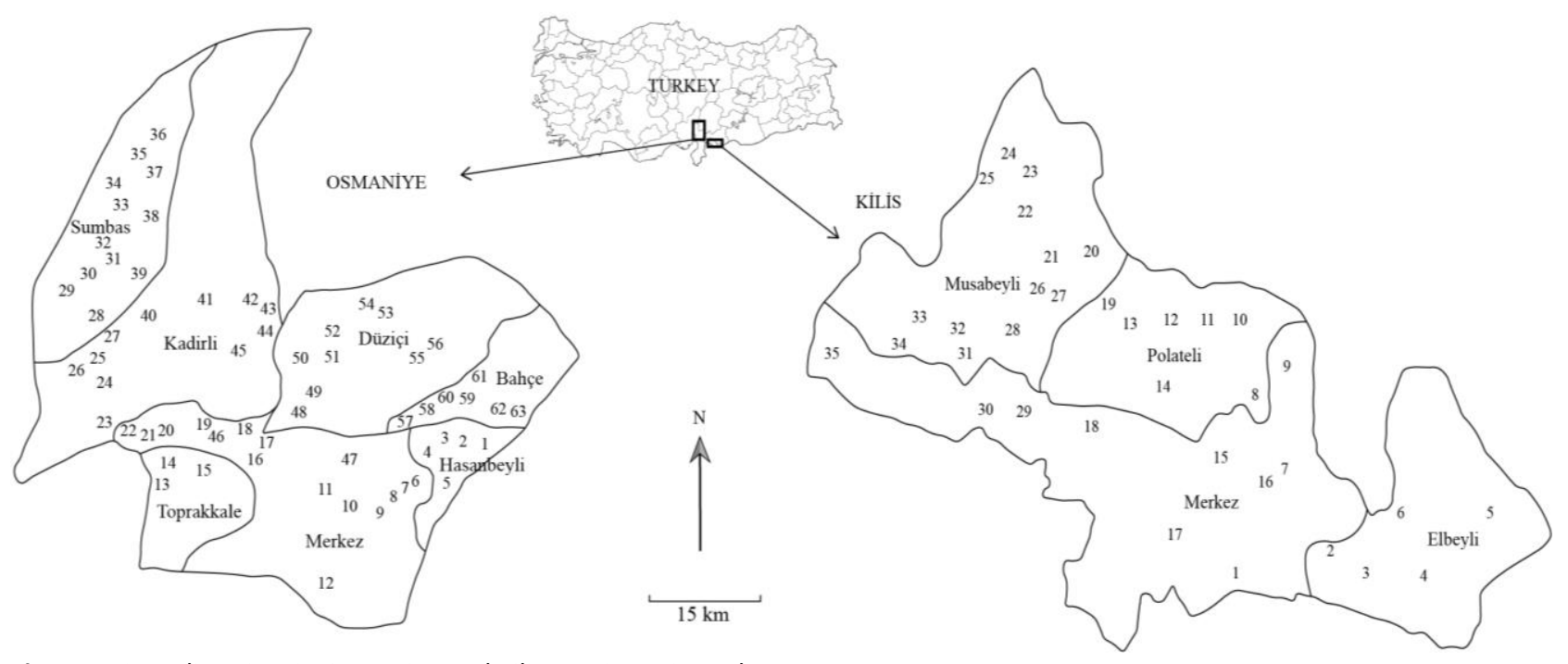

Figure 1. Sampling sites in Osmaniye and Kilis provinces in Turkey. 
further studies. Olympus $\mathrm{ACH} \quad 1 \mathrm{X}$ model stereomicroscope was used to separate ostracods from the sediment. Adult specimens were dissected with fine needles and soft body parts were separated from the valves. Olympus BX-51 model compound microscope was used for species identification. Meisch (2000) was the main source for taxonomic classification and identification but additional taxonomic sources (Bronstein, 1947; Karanovic, 2012) were used when necessary. All ostracod samples with catalog numbers are kept at the Limnology Laboratory of Bolu Abant Izzet Baysal University, Bolu, Turkey and are available upon request.

\section{Statistical Analyses}

The following statistical analyses were used for each of the aims and hypothesis stated above. We first used Shannon-Wiener Diversity index to find out species alpha diversity in different habitat types. In order to understand species occurrence patterns within 10 different habitats, Coleman heterogeneity test (Coleman, 1981; Coleman et al., 1982) was applied for the ostracods found during this study. Accordingly, if the expected curve increases sharply from the origin, heterogeneity of habitats is greater than the expected by random sampling only (Coleman, 1981; Coleman et al., 1982; Colwell and Coddington, 1994). Both analyses were done in Species Diversity and Richness program version 4.0 (Seaby and Henderson, 2006). Ostracods cooccurrence patterns were calculated with the probabilistic model (Probabilistic Species Co-occurrence Analysis in R packages) (Veech, 2013, 2014; Griffith et al., 2016). Accordingly, statistically significant pairwise possibilities of positive, negative and random species cooccurrences can be calculated at 0.05 critical level. The model basically assumes that species with negative cooccurrences tend to be found together at fewer sites than the species with positive co-occurrences while random co-occurrence means that two species occur in habitats randomly of each other.

Second, clustering relationships among ostracod species was illustrated with Unweighted Pair Group Mean Averages (UPGMA) after the quantitative data was $\log (\mathrm{e})$ transformed and tested with Jaccard's Coefficient. Multivariate Statistical Package (MVSP) program version 3.1 (Kovach, 1998) was used during UPGMA application. Individual species ecological tolerance and optimum estimates for five different environmental variables clustered in UPGMA dendrogram were calculated with C2 program (Juggins, 2003). A t-test with unequal variances in Microsoft Excel 97-2003 program was used to test whether our sampling before and after noon was biased. Similarly, numbers of species with or without swimming setae on the second antenna (A2) were analyzed with a t-test in Excel program.

Canonical correspondence analysis (CCA), an ordination method, was used to show correlations between species and environmental variables. For this reason, four variables (water temperature, $\mathrm{pH}$, salinity, dissolved oxygen) and 10 species with three or more occurrences were chosen for the analyses. During CCA, rare species were eliminated to reduce arch effect. Also, variables with 10 or more of inflation factors were not included into the analyses to eliminate multicollinearity between the variables (ter Braak, 1995). CCA was tested with Monte Carlo permutation (499 permutations) in Canoco for Windows 4.5 program (ter Braak 1995). All analyses included adult ostracods while damaged individuals, juveniles, empty carapaces and valves were excluded from them as they were unidentifiable.

Table 1. Distribution of 20 species among 10 habitat types, along with numbers of habitats (NuHab), total numbers of species in each habitat type (TotSpp) and the ratio of numbers of species per habitat (Spp/Hab). Abbreviations (ordered as in the table): $\mathrm{CN}$ (Neglecandona neglecta), CV (Cypridopsis vidua), Cpu (Cypris pubera), Hba (Heterocypris barbara), HI (Heterocypris incongruens), Hsa (Heterocypris salina), IBr (Ilyocypris bradyi), ID (Ilyocypris decipiens), IG (Ilyocypris gibba), II (Ilyocypris inermis), LI (Limnocythere inopinata), PK (Physocypria kraepelini), PV (Potamocypris variegata), PVi (Potamocypris villosa), PZe (Prionocypris zenkeri), PAl (Pseudocandona albicans), PO (Psychrodromus olivaceus), TC (Trajancypris clavata), CL (Candona lindneri), PE (Paracandona euplectella). Bold and italic codes indicate species with swimming setae on second antenna, other species with lack of swimming seta, No Sites (numbers of sites), Tot Occur (total occurrences of individual species), + (species present). *shallow water body with aquatic plants where oxygen-free process dominates.

\begin{tabular}{|c|c|c|c|c|c|c|c|c|c|c|c|c|c|c|c|c|c|c|c|c|c|c|c|}
\hline Habitat type & $\mathrm{CN}$ & $C V$ & $\mathrm{CPu}$ & $\mathrm{Hba}$ & $H I$ & $H S a$ & $\mathrm{IBr}$ & ID & IG & II & $\mathrm{LI}$ & $P K$ & $P V$ & PVi & PZe & PAl & PO & $T C$ & $\mathrm{CL}$ & $\mathrm{PE}$ & NuHab & TotSpp & Spp/Hab \\
\hline Canal & & & & & 1 & & 1 & & & & & & & & & & & 1 & & & 2 & 3 & 1.5 \\
\hline Creek & 6 & & & & 5 & & 4 & & 2 & 10 & & & & & & 1 & 3 & 2 & 1 & & 18 & 10 & 0.56 \\
\hline Ditch & & & 1 & 1 & 5 & & 3 & 2 & 1 & 1 & & & & & & & & 1 & & & 8 & 8 & 1 \\
\hline Pool & & & & & & 2 & & & & 2 & & & & & & 1 & & & & & 2 & 3 & 1.5 \\
\hline Reservoir & & & & & 1 & & & 1 & & & & 1 & 1 & & & & & 1 & & & 4 & 5 & 1.25 \\
\hline River & & & & & 1 & 1 & 3 & & & 1 & & & & & & & & 1 & & & 3 & 5 & 1.7 \\
\hline Spring & & & & & & & & & & 2 & & & & & & & 1 & & & & 2 & 2 & 1 \\
\hline Stream & 3 & & & & 5 & 2 & 3 & 2 & & 3 & 1 & & 1 & 1 & 1 & & & 1 & & & 7 & 11 & 1.6 \\
\hline Trough & & & & & 4 & 1 & 2 & & & 2 & & & & & & & & & & & 7 & 4 & 0.57 \\
\hline Wetland* & & 1 & & & & & & & & & & & & & & 1 & & & & 1 & 1 & 3 & 3 \\
\hline No Sites & 2 & 1 & 1 & 1 & 7 & 4 & 6 & 3 & 2 & 7 & 1 & 1 & 2 & 1 & 1 & 3 & 2 & 6 & 1 & 1 & & & \\
\hline Tot Occur & 9 & 1 & 1 & 1 & 22 & 6 & 16 & 5 & 3 & 21 & 1 & 1 & 2 & 1 & 1 & 3 & 4 & 7 & 1 & 1 & & & \\
\hline Osmaniye & + & + & & & + & + & + & & + & + & & + & + & + & + & + & + & + & + & + & & & \\
\hline Kilis & + & & + & + & + & + & + & + & + & & + & & + & & & + & & + & & & & & \\
\hline
\end{tabular}




\section{Results}

Total of 28 (16 living) and 17 (12 living) ostracod taxa were identified from shallow waters of Osmaniye and Kilis, respectively. Eight of the total species (Neglecandona neglecta, Heterocypris incongruens, $H$. salina, Ilyocypris bradyi, I. inermis, Potamocypris variegata, Pseudocandona albicans, Trajancypris clavata) were found in both provinces (Table 1). All species reported here are new for the ostracod fauna of each of the provinces. The three most frequently occurring species $(H$. incongruens, I. inermis, I. bradyi) were found in six to seven different habitats. The numbers of species and their abundances in natural habitats (e.g., wetlands, lakes) were twice as high as in the artificial habitats (e.g., canals, troughs). Streams and creeks displayed the highest diversity with 11 and 9 ostracod species, while reservoirs and troughs exhibited 5 and 4 species, respectively.

There were no differences in the numbers of species between lentic and lotic habitats, where the numbers of species with swimming setae (10 spp.) on the second antenna were two times more prevalent than the species without seta (5 spp.) in lentic habitats. In contrast, slightly more (8 spp.) species without setae than species with setae (7 spp.) were found in lotic habitats. There were no significant differences in the means of 13 environmental variables between the two provinces ( $P=0.82$ ) (Table 2, Appendix). Cosmoecious (and/or cosmopolitan) species seem to have wide ecological tolerance ranges for different variables (Table 3). These species were apparently found more in artificial habitats (troughs, canals, ponds, reservoirs etc.) than natural habitats. The Shannon-Wiener alpha diversity index for all samples was 1.94 with relatively small error (Jackknife standard error $=0.078$ ). The Coleman curve (Figure 2) was found slightly inclined above the observed ostracod species acquisition curve pointing to some samples heterogeneity possibly due to samplings from a variety of habitats. Comparing the numbers of ostracods among the sites with or without swimming setae on $\mathrm{A} 2$ revealed no significant difference $(P=0.85)$. The only significant positive $(P<0.05)$ cooccurrence was found between $N$. neglecta and $I$. inermis while other species pairs showed random occurrence patterns. Based on species occurrences per site, 20 species were clustered into three main groups (Figure 3). Species occurrence was found not significantly related to the sampling time of the day. During this study, there was no significant difference ( $P$ $=0.53$ ) between the numbers of species collected between 06:30-11:58 a.m. and 12:05-19:52 p.m. (Figure 4). Also, numbers of sample sites did not significantly increase the numbers of species (Table 1). CCA was able to explain about $80 \%$ of correlation between 10 species and four environmental variables (water temperature, $\mathrm{pH}$, salinity, dissolved oxygen). Of which, water temperature was the most effective $(P<0.05, F=1.866)$ variable on species occurrence among others (Figure 5). CCA diagram exhibited a clear separation between two habitats (troughs and creeks).

\section{Discussion}

Until the present study, there has been no extensive work on freshwater ostracods in Osmaniye and Kilis in Turkey. Therefore, this study presents the first documented ostracod species of these provinces. Most common eight species reported in here $(N$. neglecta, $H$. incongruens, $H$. salina, I. bradyi, I. inermis, $P$. variegata, $P$. albicans, and $T$. clavata) can also be found among the species frequently reported in the literature (e.g., Benzie, 1989; Scharf and Viehberg, 2014; Uçak et al., 2014; Akdemir et al., 2016; Yavuzatmaca et

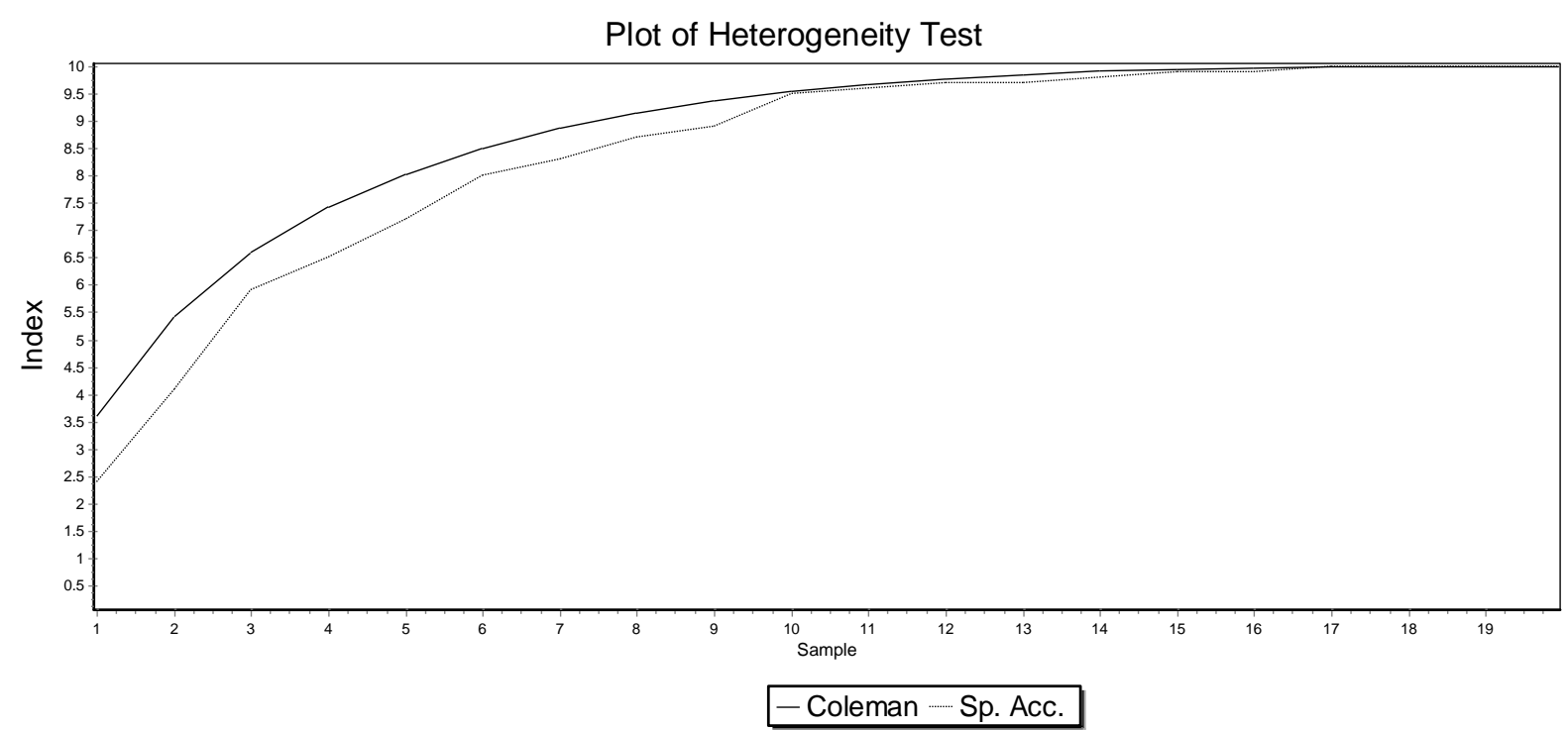

Figure 2. Coleman plot of Heterogeneity test. Coleman curve (solid line), species accumulation (Sp. Acc.) (dotted line). 
Table 2. Elevan environmental variables measured in situ from sampling sites (StNo) of Kilis and Osmaniye provinces during sampling period. Abbreviations: dissolved oxygen (DO, $\mathrm{mg} \mathrm{l}^{-1}$ ); percent oxygen saturation (DoP, \%); electrical conductivity (EC, $\mu \mathrm{S}$ $\left.\mathrm{cm}^{-1}\right)$; water temperature $\left(\mathrm{Tw},{ }^{\circ} \mathrm{C}\right)$; air temperature $\left(\mathrm{Ta},{ }^{\circ} \mathrm{C}\right)$; salinity (Sal, ppt); total dissolved solids (TDS, $\mathrm{mg} \mathrm{I}^{-1}$ ); Standard Hydrogen Electrode (SHE, $\mathrm{mV})$; air moisture (Moi, \%); air wind speed $\left(\mathrm{Wi}, \mathrm{m} \mathrm{s}^{-1}\right)$. Na, not available.

\begin{tabular}{|c|c|c|c|c|c|c|c|c|c|c|c|}
\hline StNo & $\mathrm{pH}$ & DO & DoP & $\mathrm{EC}$ & Tw & $\mathrm{Ta}$ & SHE & Sal & TDS & Moi & $\mathrm{Wi}$ \\
\hline Kilis1 & 7.59 & 8.41 & 86.1 & 785 & 16.3 & 21.2 & 253.5 & 0.47 & 611 & 40.8 & 4.3 \\
\hline Kilis2 & 9.47 & 9.81 & 126.1 & 219.7 & 28.6 & 25.5 & 192.1 & 0.1 & 135.2 & 38.6 & 3.5 \\
\hline Kilis3 & 8.24 & 8.34 & 91.3 & 540 & 19.5 & 22.5 & 221.3 & 0.29 & 636.5 & 49.7 & 10.6 \\
\hline Kilis4 & 8.09 & 6.87 & 77.3 & 732 & 21.3 & 26.9 & 232.3 & 0.39 & 513.5 & 46.2 & 1.7 \\
\hline Kilis5 & 8.23 & 12.41 & 129.1 & 795 & 17.7 & 29.2 & 243.5 & 0.46 & 604.5 & 31.3 & 7.5 \\
\hline Kilis6 & 8.07 & 7.18 & 80.6 & 594 & 21 & 27.6 & 222.6 & 0.31 & 416 & 34.7 & 2.4 \\
\hline Kilis7 & 7.92 & 6.82 & 73.9 & 615 & 19.4 & 20.8 & 226.3 & 0.34 & 448.5 & 54.2 & 22.9 \\
\hline Kilis8 & 9.32 & 11.04 & 127.9 & 437.9 & 22.8 & 20.8 & 221.8 & 0.22 & 296.4 & 48.4 & 10.3 \\
\hline Kilis9 & 9.7 & 9.97 & 120.4 & 876 & 24.6 & 25.7 & 210.5 & 0.43 & 572 & 41.3 & 2.1 \\
\hline Kilis10 & 8.29 & 8.7 & 99.3 & 926 & 21.8 & 22.4 & 223.3 & 0.49 & 643.5 & 42.3 & 8.4 \\
\hline Kilis11 & 7.72 & 6.51 & 69.7 & 448.4 & 18.6 & 22.8 & 246.6 & 0.25 & 332.15 & 48.2 & 12.9 \\
\hline Kilis12 & 8.6 & 9.69 & 106.5 & 448.5 & 20.1 & 25.3 & 238.7 & 0.24 & 321.1 & 43.1 & 5.9 \\
\hline Kilis13 & 7.99 & 5.23 & 55.7 & 342.7 & 18.6 & 27.7 & 247.4 & 0.19 & 254.15 & 41.3 & 3.3 \\
\hline Kilis14 & 7.91 & 9.26 & 106.7 & 479.4 & 22.1 & 26.3 & 286.5 & 0.24 & 329.55 & 41.5 & 2 \\
\hline Kilis15 & 8.3 & 8.23 & 89.9 & 522 & 19.6 & 23.7 & 272.9 & 0.28 & 377 & 43.4 & 13.2 \\
\hline Kilis16 & 8.64 & 6.92 & 79.8 & 371.8 & 22.3 & 23.5 & 266.2 & 0.19 & 254.8 & 44.2 & 5.7 \\
\hline Kilis17 & 7.43 & 4.85 & 50.7 & 626 & 17.7 & 24.2 & 261.9 & 0.36 & 474.5 & 42.5 & 2.2 \\
\hline Kilis18 & 7.97 & 7.93 & 81.4 & 579 & 17.3 & 16.7 & 259.9 & 0.34 & 448.5 & 57.7 & 4.2 \\
\hline Kilis19 & 7.11 & 4.95 & 51.3 & 567 & 16.9 & 17.5 & 251.9 & 0.33 & 435.5 & 66.4 & 1.4 \\
\hline Kilis20 & 8.35 & 7.58 & 74.6 & 376.1 & 14.7 & 15.8 & 255.3 & 0.23 & 303.55 & 67.3 & 2.3 \\
\hline Kilis21 & 8.48 & 8.45 & 83.6 & 517 & 14.7 & 18.6 & 267 & 0.31 & 416 & 62.3 & 2.1 \\
\hline Kilis22 & 8.21 & 8.2 & 81.9 & 491.9 & 15.1 & 24.8 & 267.2 & 0.3 & 393.25 & 46.2 & 2.3 \\
\hline Kilis23 & 9.6 & 7.7 & 81.7 & 213.4 & 18.3 & 26.6 & 245 & 0.12 & 159.25 & 38.4 & 2.4 \\
\hline Kilis24 & 8.21 & 8.36 & 84.2 & 512 & 15.8 & 25.5 & 247.6 & 0.3 & 409.5 & $\mathrm{Na}$ & 3.6 \\
\hline Kilis25 & 7.74 & 5.6 & 56 & 594 & 15.3 & 26.9 & 264.9 & 0.36 & 474.5 & 36.3 & 8.2 \\
\hline Kilis26 & 8.46 & 7.11 & 78.3 & 453 & 20.1 & 28.1 & 248.7 & 0.24 & 324.35 & 34.3 & 1.5 \\
\hline Kilis27 & 8.41 & 6.85 & 75.4 & 449.4 & 20 & 24.4 & 244.5 & 0.24 & 322.05 & 34.9 & 4.2 \\
\hline Kilis28 & 7.34 & 5.71 & 50.7 & 747 & 18.5 & 26.4 & 264.3 & 0.42 & 552.5 & 46.8 & 6.2 \\
\hline Kilis29 & 8.47 & 11.7 & 132.1 & 458.3 & 21.2 & 32.5 & 257.2 & 0.24 & 321.1 & 36.3 & 4.5 \\
\hline Kilis30 & 8.76 & 8.4 & 93.6 & 786 & 20.8 & 27.9 & 244.1 & 0.42 & 559 & 36.1 & 19.3 \\
\hline Kilis31 & 8.43 & 6.25 & 71.9 & 431.3 & 22.5 & 27.6 & 246.8 & 0.22 & 295.1 & 28.1 & 3.2 \\
\hline Kilis32 & 8.59 & 8.03 & 90.7 & 669 & 20.9 & 26.3 & 258.6 & 0.35 & 474.5 & 33.2 & 4.2 \\
\hline Kilis33 & 8.67 & 8.6 & 98.1 & 660 & 21.8 & 26.3 & 254.9 & 0.34 & 455 & 30.9 & 7.5 \\
\hline Kilis34 & 8.76 & 6.04 & 69.1 & 488.5 & 22.1 & 25.5 & 238.9 & 0.25 & 335.4 & 34.3 & 8.7 \\
\hline Kilis35 & 8.53 & 8.13 & 87.4 & 1013 & 18.7 & 27.1 & 247.1 & 0.57 & 747.5 & 32.5 & 5.2 \\
\hline Osmaniye1 & 7.33 & 6.02 & 64.9 & 528 & 18.6 & 22.5 & 230.1 & 0.29 & 390 & 44.4 & 0.29 \\
\hline Osmaniye2 & 7.45 & 7.58 & 76.3 & 570 & 15.5 & 27.7 & 259.2 & 0.34 & 455 & 36.6 & 0.34 \\
\hline Osmaniye3 & 7.56 & 6.32 & 63.2 & 559 & 15.3 & 23.3 & 276.4 & 0.34 & 448.5 & 40.1 & 0.34 \\
\hline Osmaniye4 & 8.46 & 8.5 & 86.5 & 497.7 & 16.2 & 25 & 311 & 0.29 & 388.7 & 43.1 & 0.29 \\
\hline Osmaniye5 & 8.74 & 8.21 & 85.9 & 754 & 17.1 & 26.4 & 282.1 & 0.44 & 578.5 & 36.1 & 0.44 \\
\hline Osmaniye6 & 8.14 & 8.53 & 82.5 & 613 & 13.7 & 22.5 & 273.3 & 0.29 & 507 & 39.7 & 0.29 \\
\hline Osmaniye7 & 7.89 & 7.9 & 77.2 & 262.8 & 14.3 & 23.7 & 278.1 & 0.16 & 214.5 & 43.4 & 0.16 \\
\hline Osmaniye8 & 8.08 & 8.05 & 78.8 & 292.1 & 14.6 & 21.4 & 280.3 & 0.18 & 236.6 & 41.7 & 0.18 \\
\hline Osmaniye9 & 8.5 & 8.2 & 80.2 & 351 & 14.3 & 24.8 & 276.7 & 0.21 & 286.65 & 41.2 & 0.21 \\
\hline Osmaniye10 & 8.72 & 9.14 & 87 & 421.9 & 13 & 22.9 & 280.4 & 0.27 & 356.2 & 53.8 & 0.27 \\
\hline Osmaniye11 & 8.69 & 8.92 & 93.4 & 277.4 & 17.8 & 21.7 & 270.8 & 0.15 & 208 & 63.7 & 0.15 \\
\hline Osmaniye12 & 8.03 & 8.41 & 76.3 & 186.7 & 10.8 & 17.3 & 277.7 & 0.12 & 167.05 & 56.3 & 0.12 \\
\hline Osmaniye13 & 7.81 & 6.31 & 62.1 & 417.2 & 14.8 & 22.1 & 261.3 & 0.25 & 337.35 & 60.3 & 0.25 \\
\hline Osmaniye14 & 7.8 & 5.19 & 52.2 & 460.5 & 15.7 & 22.5 & 269.9 & 0.27 & 364 & 65.5 & 0.27 \\
\hline Osmaniye15 & 8.03 & 4.8 & 49.6 & 568 & 16.9 & 24 & 262.7 & 0.53 & 435.5 & 54.9 & 0.53 \\
\hline Osmaniye16 & 7.84 & 6.52 & 62.8 & 384 & 13.3 & 26.2 & 273.3 & 0.24 & 321.75 & 55.4 & 0.24 \\
\hline Osmaniye17 & 8.12 & 6.68 & 74.5 & 1007 & 20.6 & 28.2 & 273.4 & 0.55 & 715 & 45.3 & 0.55 \\
\hline Osmaniye18 & 8.35 & 7.95 & 82.2 & 1327 & 17 & 27.7 & 264.9 & 0.79 & 1020.5 & 50.6 & 0.79 \\
\hline Osmaniye19 & 6.93 & 4.3 & 47 & 748 & 19.6 & 25.2 & 267.8 & 0.41 & 539.5 & 56.9 & 0.41 \\
\hline Osmaniye20 & 7.38 & 3.08 & 32.2 & 563 & 18.3 & 31.2 & 265.5 & 0.32 & 422.5 & 48.6 & 0.32 \\
\hline Osmaniye21 & 7.7 & 5.61 & 60.8 & 599 & 19.1 & 31.2 & 269.1 & 0.33 & 436.5 & 43.9 & 0.33 \\
\hline Osmaniye22 & 7.9 & 8.67 & 84.1 & 400.8 & 14.3 & 28.3 & 283.5 & 0.24 & 327.6 & 48.9 & 0.24 \\
\hline Osmaniye23 & 8.14 & 9.13 & 93.3 & 428.4 & 16.2 & 28.9 & 298.1 & 0.25 & 334.75 & 50.3 & 0.25 \\
\hline Osmaniye24 & 7.9 & 4.32 & 48.8 & 508 & 21.5 & 27.1 & 282.2 & 0.26 & 357.5 & 49.2 & 0.26 \\
\hline Osmaniye25 & 8.75 & 10.8 & 142.1 & 828 & 20.2 & 33 & 225.4 & 0.37 & 494 & 39.3 & 0.37 \\
\hline
\end{tabular}


Table 2. Continued

\begin{tabular}{|c|c|c|c|c|c|c|c|c|c|c|c|}
\hline StNo & $\mathrm{pH}$ & DO & DoP & $\mathrm{EC}$ & Tw & $\mathrm{Ta}$ & SHE & Sal & TDS & Moi & Wi \\
\hline Osmaniye26 & 7.71 & 4.91 & 55.9 & 731 & 21.8 & 31.8 & 255.9 & 0.38 & 507 & 45.2 & 0.38 \\
\hline Osmaniye27 & 7.92 & 7.44 & 86.0 & 778 & 21.9 & 30 & 233.1 & 0.41 & $\mathrm{Na}$ & 39.6 & 0.41 \\
\hline Osmaniye28 & 8.41 & 8.46 & 93.2 & 431.9 & 20.3 & 32.1 & 262.7 & 0.23 & 308.1 & 36.6 & 0.23 \\
\hline Osmaniye29 & 8.3 & 8.75 & 100.3 & 593 & 19.5 & 35.2 & 249.4 & 0.32 & 429 & 36.7 & 0.32 \\
\hline Osmaniye30 & 8.46 & 8.04 & 105 & 580 & 33.3 & 30.2 & 196.1 & 0.24 & 325 & 43.6 & 0.24 \\
\hline Osmaniye31 & $\mathrm{Na}$ & 7.21 & 78.1 & 651 & 19 & 34 & 248.6 & 0.36 & 481 & 48.6 & 0.36 \\
\hline Osmaniye32 & 8.19 & 1.52 & 22.3 & 199.7 & 31.1 & 34.3 & 204.2 & 0.08 & 116.35 & 43.5 & 0.08 \\
\hline Osmaniye33 & 8.08 & 6.33 & 73.6 & 338 & 23.1 & 32.4 & 225.3 & 0.25 & 338 & 45.5 & 0.25 \\
\hline Osmaniye34 & 7.11 & 5.00 & 49.2 & 586 & 14.5 & 32.8 & 261.1 & 0.36 & 250.9 & 40.8 & 0.36 \\
\hline Osmaniye35 & 8.28 & 8.43 & 83.9 & 329.7 & 15.1 & 27.8 & 273.1 & 0.2 & 264.55 & 48.3 & 0.2 \\
\hline Osmaniye36 & 7.96 & 6.25 & 64.4 & 479.5 & 16.5 & 27.5 & 269.9 & 0.28 & 371.8 & 44.1 & 0.28 \\
\hline Osmaniye37 & 8.31 & 4.89 & 52.5 & 364.2 & 18.6 & 21.3 & 228.6 & 0.2 & 269.75 & 57.3 & 0.2 \\
\hline Osmaniye38 & 7.79 & 6.29 & 63.0 & 728 & 15.5 & 27.5 & 238.5 & 0.44 & 578.5 & 50.1 & 0.44 \\
\hline Osmaniye39 & 7.81 & 5.09 & 59.7 & 361.6 & 22.6 & 28.4 & 241.9 & $\mathrm{Na}$ & 247 & 51.3 & $\mathrm{Na}$ \\
\hline Osmaniye40 & 8.09 & 8.62 & 88.5 & 508 & 16.6 & 23.4 & 269.7 & 0.3 & 396.5 & 66 & 0.3 \\
\hline Osmaniye41 & 8.46 & 4.66 & 49.8 & 137.4 & 18.8 & 24.4 & 246.5 & 0.07 & 101.4 & 58.7 & 0.07 \\
\hline Osmaniye42 & 8.25 & 8.94 & 91.4 & 520 & 16.4 & 24.4 & 274.6 & 0.3 & 403 & 61.2 & 0.3 \\
\hline Osmaniye43 & 8.51 & 7.02 & 82.9 & 382.5 & 23.7 & 28.5 & 251.5 & 0.19 & 254.8 & 56.3 & 0.19 \\
\hline Osmaniye44 & 8.69 & 8.41 & 97.8 & 380.9 & 23 & 27.6 & 251.8 & 0.19 & 257.4 & 54.9 & 0.19 \\
\hline Osmaniye45 & 8.34 & 8.51 & 88.2 & 412.6 & 22.9 & 29.2 & 239.3 & 0.21 & 279.5 & 57.6 & 0.21 \\
\hline Osmaniye46 & 7.34 & 8.42 & 88.6 & 893 & 17.7 & 26.8 & 290.3 & 0.52 & 676 & 55.5 & 0.52 \\
\hline Osmaniye47 & 8.81 & 8.63 & 97.2 & 496.7 & 20.8 & 30.6 & 279.4 & 0.26 & 351 & 42.2 & 0.26 \\
\hline Osmaniye48 & 7.55 & 3.43 & 37.3 & 739 & 19.6 & 32.7 & 278 & 0.41 & 539.5 & 46.3 & 0.41 \\
\hline Osmaniye49 & 9.78 & 8.95 & 119.2 & 234.3 & 30.4 & 32.5 & 223.3 & 0.1 & 137.8 & 42.4 & 0.1 \\
\hline Osmaniye50 & 6.99 & 6.44 & 67.4 & 708 & 18.1 & 31.6 & 286.1 & 0.4 & 533 & 44.5 & 0.4 \\
\hline Osmaniye51 & 7.93 & 3.82 & 46.7 & 283.4 & 25.3 & 34.2 & 262.8 & 0.13 & 183.3 & 42.6 & 0.13 \\
\hline Osmaniye52 & 8.01 & 8.05 & 88.9 & 830 & 20.3 & 35.2 & 283.2 & 0.45 & 591.5 & 44.5 & 0.45 \\
\hline Osmaniye53 & 7.68 & 4.92 & 57.9 & 602 & 23.5 & 36.5 & 265.1 & 0.3 & 403 & 36.9 & 0.3 \\
\hline Osmaniye54 & 9.65 & 8.43 & 126.1 & 141.2 & 36.2 & 35.7 & 211.6 & 0.05 & 76.05 & 36.8 & 0.05 \\
\hline Osmaniye55 & 7.92 & 6.17 & 65.5 & 301.5 & 18.6 & 35.4 & 205.8 & 0.16 & 223.6 & 30.1 & 0.16 \\
\hline Osmaniye56 & 8.7 & 9.12 & 93.5 & 404 & 17 & 36.9 & 245.1 & 0.23 & 310.05 & 30.3 & 0.23 \\
\hline Osmaniye57 & $\mathrm{Na}$ & 7.15 & 77 & 581 & 19.1 & 34.3 & 266.2 & 0.32 & 422.5 & 36.2 & 0.32 \\
\hline Osmaniye58 & 7.57 & 6.96 & 71.2 & 614 & 16.3 & 33.3 & 277.9 & 0.36 & 481 & 36.2 & 0.36 \\
\hline Osmaniye59 & 8.41 & 9.03 & 93.3 & 306 & 14 & 26.5 & 279.9 & 0.19 & 251.55 & 48 & 0.19 \\
\hline Osmaniye60 & 8.01 & 6.1 & 58.9 & 405.1 & 13.9 & 28.1 & 283.5 & 0.25 & 334.7 & 40.1 & 0.25 \\
\hline Osmaniye61 & 8.59 & 8.56 & 85.1 & 343 & 15.5 & 29.5 & 286 & 0.2 & 272.35 & 48.1 & 0.2 \\
\hline Osmaniye62 & 8.06 & 7.68 & 81.6 & 171.7 & 18.1 & 27.9 & 281.4 & 0.09 & 128.7 & 43.8 & 0.09 \\
\hline Osmaniye63 & 7.8 & 7.5 & 77.9 & 151.9 & 17.1 & 25.6 & 278.3 & 0.08 & 116.35 & 50.1 & 0.08 \\
\hline
\end{tabular}

al., 2017). Indeed, finding a positive co-occurrence between the two of the species ( $N$. neglecta and $I$. inermis) also suggests that such species seem to have similar habitat preferences and ecological conditions. For example, both species lack of swimming setae and are bottom dependent. This is probably one of the reasons of their occurrence in the third group of clustering analyses (Figure 3) where four of six species (including $N$. neglecta and $I$. bradyi) are known without swimming setae. Similar results can be seen in the other two clustering groups. In the second group, five of six species are known with swimming setae while two of three species without setae are clustered in the first group. As seen, none of the groups covers solely the species with or without swimming setae. Accordingly, this gives an impression that some other factors effective on species co-occurrence patterns. Moreover, contribution of these species into the ostracod diversity is valuable and is of great importance since these species were found in almost all habitats (Table 1). Species diversity and numbers of species of Osmaniye and Kilis are lower than many of the other provinces (or cities) in Turkey (Table 4); for instance, Gaziantep province located between these two provinces includes 29 ostracod species from 57 sites (Akdemir et al., 2016) while three other provinces (Bartın with 13, Düzce with 19 and Zonguldak with 18 species) consist of higher numbers of species. In contrast, the ratios (numbers of species per site and surface area) in Osmaniye and Kilis are relatively higher than some of the others (Table 4). One may argue that different factors (e.g., environmental heterogeneity, sampling effort) may cause such differences in the numbers of species among the provinces. However, similarities in the sampling methods and types of habitats (cf. Uçak et al., 2014; Akdemir et al., 2016; Külköylüoğlu et al., 2016; Yavuzatmaca et al., 2017) facilitate effects of the differences. Thereby, we are confident for comparisons of the numbers of species made among the provinces. 


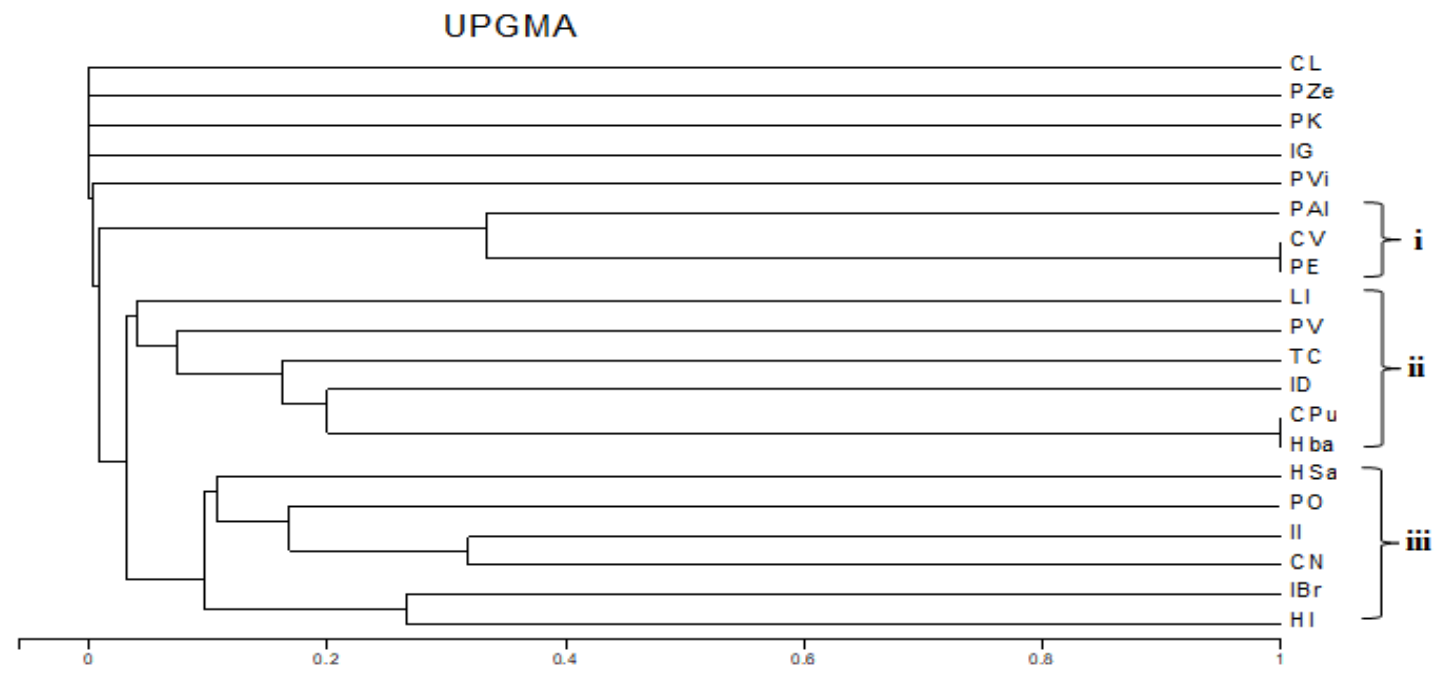

Jaccard's Coefficient - Data $\log (e)$ transformed

Figure 3. Clustering relationship of UPGMA for 20 species found from Osmaniye and Kilis provinces in Turkey. Three (i, ii, iii) groups are clustered based on species occurrences in different habitats. See Table 1 for abbreviations.

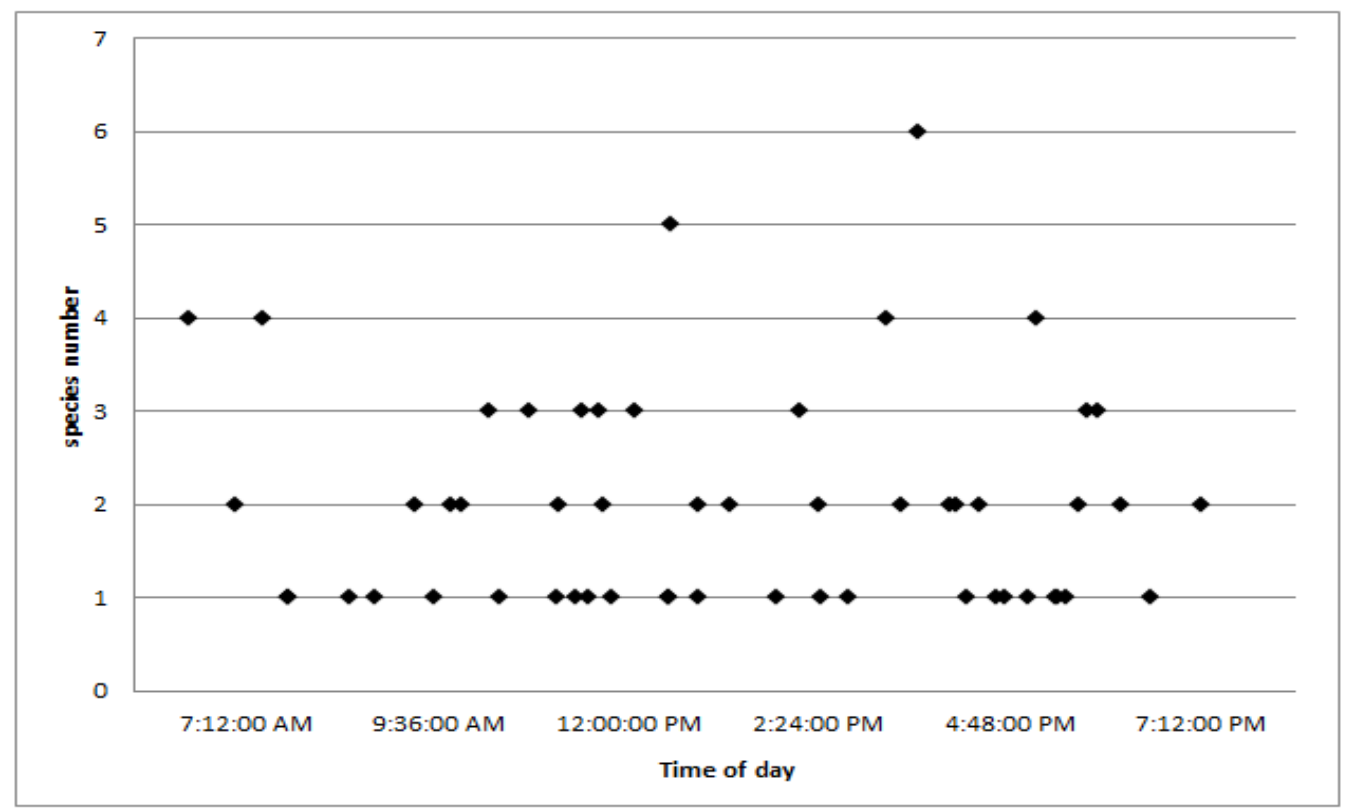

Figure 4. Numbers of species (diamonds) occurrences during a sampling day from 06:30 a.m. to 19:52 p.m. Note that dividing the data at noon was due to (1) make a proper comparison between our data and data in Ganning (1967) who used similar way to compare ostracod occurrences, and (2) possibility of about equal time during our sampling hours in a day in May 2015.

\section{Abiotic Factors and Habitat Preferences}

Several abiotic factors could result in lower diversity findings within this study such as sampling time, sampling frequency (i.e., one or more time of sampling) within a short period of time, seasonal differences, and geographical factors. The roles of biotic factors are out of the scope of this study and not published here. From the results of the present study, one might suggest that ostracod diversity might substantially be correlated to the habitat suitability rather than habitat heterogeneity or habitat type. In our view, rather than microhabitat type, conditions in that microhabitats appear to be more effective on many (if not all) freshwater ostracods. This is because a species (e.g., those cosmopolitan species stated above) can be found in many different aquatic habitats in different geographic region of the world. Indeed, supportive evidence on our view comes from previous studies. For example, Benzie (1989) reported that numbers of ostracod species (and diversity) were correlated with increasing numbers of detritus type in a coastal sanddune lake, Loch of Strathbeg, Scotland. The author observed an increase in ostracod diversity and species 
numbers from open sandy sites to emergent macrophyte habitats in the littoral zone. Results of his study concentrated on the importance of microhabitat preferences within the same habitat (i.e. lake) by ostracods. He concluded that occurrence patterns of some species such as Cypridopsis vidua and Herpetocypris reptans were corresponded to the presence of the aquatic plant Eleocharis. In contrast, other species (Cypria ophtalmica, C. exculpta, Candona candida) found in the same habitat preferred another aquatic plant Phragmites while Limnocythere inopinata was correlated to sandy substrates. Although Benzie's work focused on the microhabitat preferences, his results may be used to explain ostracods occurrences amid (macro)habitats. Indeed, recent studies underlined that importance of habitat type. For example, three different habitat types (limnocrene spring, pond and stream) were characterized by high ostracod diversities in Kahramanmaraş province (Turkey) (Külköylüoğlu et al., 2012b). In contrast, Yavuzatmaca et al. (2015) reported only four frequently occurring species ( $N$. neglecta, $\mathrm{H}$. incongruens, I. bradyi and Psychrodromus olivaceus) mostly common in spring, creek and ponds of Adıyaman province (Turkey).

\section{Swimming vs Non-Swimming Ability}

Similarly, as mentioned above, Akdemir et al. (2016) underlined that some ostracod species collected from a variety of habitats in Gaziantep showed speciesspecific habitat preferences depending on the presence and absence of swimming setae. Moreover, these authors found that species without swimming setae on the second antenna seemed to prefer lotic (flowing)

Table 3. Ecological tolerance (Tol) and optimum (Opt) values of 10 most frequently occurring species and five environmental variables measured during this study. Abbreviations: Numbers of species occurrence (Count), maximum numbers of individuals (Max), Hill's coefficient or measure of effective number of occurrences (N2), dissolved oxygen (DO, mg l-1 $)$, electrical conductivity $\left(\mathrm{EC}, \mu \mathrm{S} \mathrm{cm}{ }^{-1}\right)$, water temperature $\left(\mathrm{Tw},{ }^{\circ} \mathrm{C}\right)$, elevation $(\mathrm{Elev}, \mathrm{m})$. Note that one time occurrences under Count are only given here to compare the mean values

\begin{tabular}{|c|c|c|c|c|c|c|c|c|c|c|c|c|c|}
\hline \multirow[b]{2}{*}{ Species } & \multirow[b]{2}{*}{ Count } & \multirow[b]{2}{*}{ Max } & \multirow[b]{2}{*}{ N2 } & \multicolumn{2}{|c|}{ pH } & \multicolumn{2}{|c|}{ DO } & \multicolumn{2}{|c|}{ EC } & \multicolumn{2}{|c|}{ Tw } & \multicolumn{2}{|c|}{ Elev } \\
\hline & & & & Opt & Tol & Opt & Tol & Opt & Tol & Opt & Tol & Opt & Tol \\
\hline H. incongruens & 22 & 93 & 6.98 & 8.1 & 0.63 & 8.7 & 2.02 & 656 & 201 & 18.8 & 2.93 & 505 & 218 \\
\hline I. inermis & 21 & 84 & 5.78 & 7.55 & 0.58 & 5.9 & 1.45 & 539 & 141 & 16.7 & 2.41 & 640 & 191 \\
\hline I. bradyi & 16 & 35 & 6.02 & 7.94 & 0.3 & 6.9 & 1.58 & 649 & 153 & 19.8 & 2.68 & 263 & 256 \\
\hline N. neglecta & 8 & 6 & 6.31 & 8.16 & 0.31 & 7.9 & 1.7 & 417 & 106 & 17.6 & 2.76 & 733 & 197 \\
\hline T. clavata & 7 & 2 & 6.25 & 8.53 & 0.74 & 8.3 & 2.17 & 656 & 187 & 21.1 & 2.7 & 507 & 248 \\
\hline H. salina & 7 & 82 & 2.17 & 8.06 & 0.59 & 8.6 & 1.02 & 866 & 179 & 20.6 & 2.45 & 615 & 288 \\
\hline I. decipiens & 5 & 16 & 3.69 & 8.59 & 0.9 & 7.9 & 1.67 & 580 & 223 & 21 & 2.84 & 647 & 61 \\
\hline P. olivaceus & 4 & 9 & 2.42 & 8.07 & 0.18 & 7.4 & 1.06 & 524 & 207 & 17.1 & 3.1 & 475 & 549 \\
\hline I. gibba & 3 & 56 & 1.15 & 9.65 & 1.34 & 8.8 & 2.78 & 261 & 288 & 29.6 & 8.77 & 311 & 68 \\
\hline P. albicans & 3 & 2 & 3 & 8.21 & 0.72 & 6.6 & 3.04 & 631 & 59 & 20.3 & 1.82 & 429 & 337 \\
\hline Mean & 9.6 & 38.5 & 4.38 & 8.29 & 0.63 & 7.7 & 1.85 & 578 & 174 & 20.3 & 3.25 & 513 & 241 \\
\hline Max & 22 & 93 & 6.98 & 9.65 & 1.34 & 8.8 & 3.04 & 866 & 288 & 29.6 & 8.77 & 733 & 549 \\
\hline Min & 3 & 2 & 1.15 & 7.55 & 0.18 & 5.9 & 1.02 & 261 & 59 & 16.7 & 1.82 & 263 & 61 \\
\hline
\end{tabular}

Table 4. Comparison of numbers of species (Num.Spp) and numbers of sampling sites (Num.Site) from 13 provinces with different surface area (Area, $\mathrm{km}^{2}$ ) and the ratio of species per sampled site within the province (Spp/site) and per square kilometer (Spp/ $\left./ \mathrm{km}^{2}\right)$. Sources: a, Yavuzatmaca et al., 2015; b, Külköylüoğlu et al., 2017a; c, Külköylüoğlu et al., 2016; d, Külköylüoğlu et al., 2012d; e, Külköylüoğlu et al., 2012b; f, Külköylüoğlu et al., 2012c; g, Uçak et al., 2014; h, Külköylüoğlu et al., 2017b; i, Akdemir et al., 2016; j, this study

\begin{tabular}{lcccccc}
\hline Name & Area & Num.Spp & Num.Site & Spp/site & Spp/ $/ \mathrm{km}^{2}$ & Source \\
\hline Adıyaman & 7164 & 27 & 111 & 0.2432 & 0.0038 & $\mathrm{a}$ \\
Bartın & 2079 & 13 & 27 & 0.4815 & 0.0063 & $\mathrm{~b}$ \\
Zonguldak & 3306 & 18 & 42 & 0.4286 & 0.0054 & $\mathrm{~b}$ \\
Çankırı & 7388 & 25 & 114 & 0.2193 & 0.0034 & $\mathrm{c}$ \\
Diyarbakır & 15272 & 23 & 48 & 0.4792 & 0.0015 & $\mathrm{~d}$ \\
K. Maraş & 7173 & 30 & 68 & 0.4412 & 0.0042 & $\mathrm{e}$ \\
Van & 10115 & 29 & 57 & 0.5088 & 0.0029 & $\mathrm{f}$ \\
Ankara & 25437 & 29 & 152 & 0.1908 & 0.0011 & $\mathrm{~g}$ \\
Karabük & 4145 & 22 & 75 & 0.2933 & 0.0053 & $\mathrm{~h}$ \\
Düzce & 2573 & 19 & 73 & 0.2603 & 0.0074 & $\mathrm{~h}$ \\
Antep & 6222 & 29 & 57 & 0.5088 & 0.0047 & $\mathrm{i}$ \\
Kilis & 1642 & 12 & 22 & 0.5455 & 0.0073 & $\mathrm{j}$ \\
Osmaniye & 3280 & 16 & 32 & 0.5 & 0.0049 & $\mathrm{j}$ \\
\hline
\end{tabular}


habitats more than lentic (standing) aquatic habitats. Despite this preference, there was no significant difference in the numbers of species with or without setae both in lotic and in lentic waters (Akdemir et al., 2016). Although there were no differences in the numbers of species between lentic and lotic habitats (15 species in each) in the present study, numbers of species with swimming setae (10 spp.) were two times more prevalent than the species without seta ( $5 \mathrm{spp}$.) in lentic habitats. Results of CCA diagram (Figure 5A) supports the findings of Akdemir et al. (2016) where troughs (artificial habitats) and creeks (natural habitats) sites can be partially separated from each other. This is proably due to differences in physico-chemical characteristics of those habitats, indicating that species with swimming ability are generally located in troughs when nonswimmers are placed in creeks, representing the flowing habitats (Figure 5B). In Hoff's (1942) study on a variety of aquatic bodies of Illinois, many ostracod species were found in lotic (he used the term "running") waters without swimming ability while many others in lentic (he used the term "quiet") waters were able to swim with their well-developed swimming setae. In another study, working with many sampling sites (> 500 sites) in south-central Texas, Wise (1961) compared numbers of ostracods between lentic and lotic waters where he found about the same number of species (8 and 10 spp., respectively). Wise (1961) did not discuss presence or absences of setae on ostracods. However, we were able to compare numbers of swimmer and nonswimmer species from his list and tables, and found the same numbers of species with or without setae ( 5 and 5 spp.) in lotic waters while number of species with setae (7 spp.) was much higher than species with no setae (1 sp.) in lentic waters. These results correspond with the results of the present study although numbers of species found in each habitat are different.

These results, as clearly pointed out by Akdemir et al. (2016) (but also see Hoff, 1942; Wise, 1961; McLay, 1978a, b; Külköylüoğlu et al., 2012b; Yavuzatmaca et al., 2015), suggest that species with setae in lentic waters have an advantage over non-swimmers (lacking setae) during active dispersion or migration among the sites or habitats. Active dispersion with setae can also reduce the competitive interactions within the same habitat by means of occupying different niches (McLay, 1978a, b). On the other hand, species without setae are bottom dependent and possibly take advantage of flowing waters during passive dispersion. In both cases, species do increase their dispersion abilities and their adaptive values in the habitats. One possible question herein can be, "What makes these species so successful amid a variety of habitats?" Although there is no clear answer for this kind of question at the moment due to lack of information about specific species ecology, it can be considered that species with high levels of tolerances to different environmental variables seem to survive in a wide range of habitats. Indeed, the authors of the previous studies mentioned above clearly underlined that these species showed high tendencies to have wider tolerance ranges than those of non-cosmopolitan species. Our results (Table 3) support these findings but it is important to point out that species with wide tolerance levels generally tend to reduce their optimum
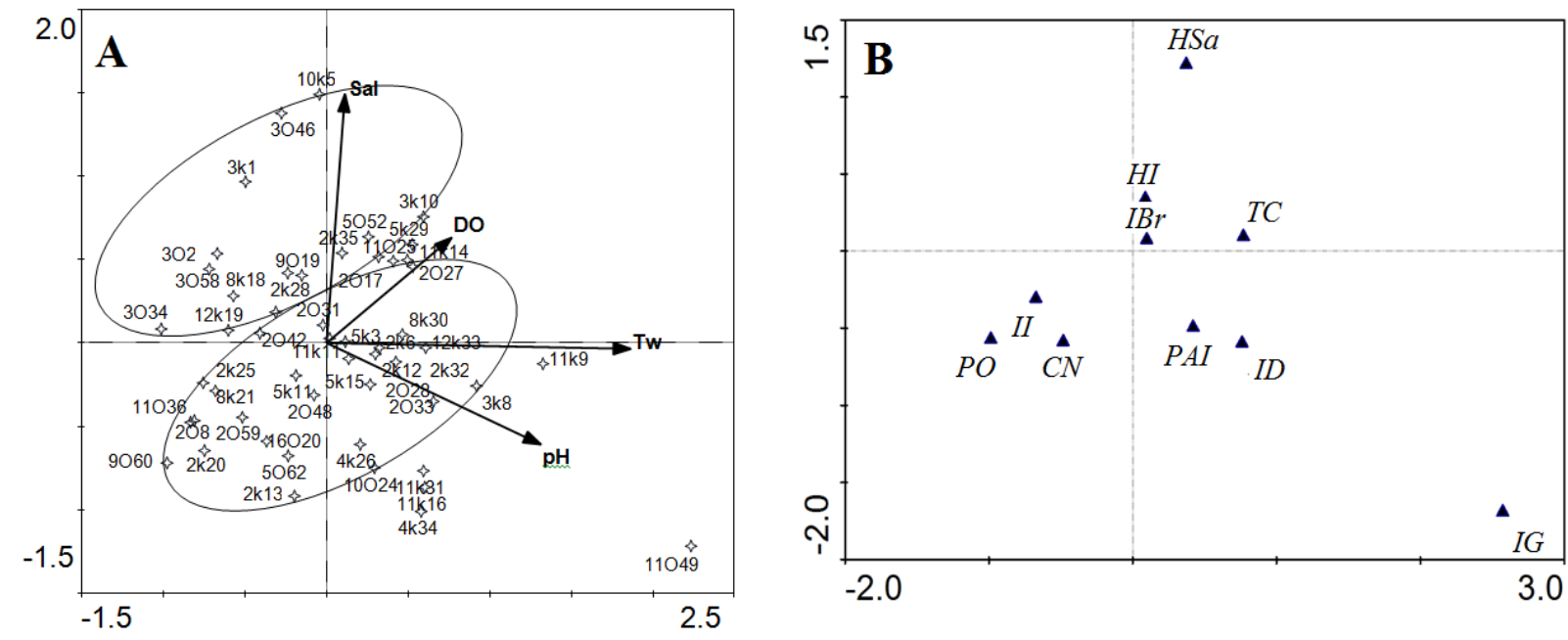

Figure 5. CCA diagrams show (A) four environmental variables and 50 sampling sites, and (B) distribution of 10 species collected from three or more different sampling sites. Two habitat types (troughs and creeks) were separated at upper and lower ellipses, respectively. Note to the coding in $(A)$ where the first one or two numbers indicate habitat type, the letter ( $O$ or $k$ ) in the middle represents Osmaniye or Kilis, and the last one or two numbers show the samling site. For example, 3046 should be read as 3 (habitat type, lake), O (Osmaniye), and 46 (sampling site number). Habitat types: 2) creek, 3) trough, 4) reservoir, 5) stream, 6) well, 8) river, 9) spring water, 10) canal, 11) water body, 12) pool, 16) wetland. Abbreviations: CN (Neglecandona neglecta), HI (Heterocypris incongruens), HSa (Heterocypris salina), IBr (Ilyocypris bradyi), ID (Ilyocypris decipiens), IG (Ilyocypris gibba), II (Ilyocypris inermis), PAI (Pseudocandona albicans), PO (Psychrodromus olivaceus), TC (Trajancypris clavata), Sal (salinity), DO (dissolved oxygen) and Tw (water temperature). 
values. Lowering the optimum values seems to aid species to obtain minimum conditions that they can survive while they increase their tolerance ranges. Having wide ranges of tolerances can also help species co-occurrence patterns with others, possibly, reducing the effect of competitive interactions. Populations of species (including humans) with such adaptive values apparently spread around widely and possibly increase their survival chance more than those species with high optimum (and lower tolerance) ranges. In this case, they can better adapt to new conditions within and/or among the habitats. Thus, previous results are in general agreement with the present study, in which streams and creeks had highest diversity with 11 and 9 ostracods when reservoirs and troughs exhibited 5 and 4 species.

\section{Sampling Time and Occurrence Pattern}

In terms of the sampling time, as stated above, one of the earliest studies by Ganning (1967) on H. salina found that species abundance and occurrences were higher early in the morning (04:00 a.m.) than afternoon (16:00 p.m.) because $H$. salina showed negative response to light intensities. In another study, Benzie (1984) reported a series of observations on the diurnal migratory behavior of $H$. incongruens from 08:00 a.m. to 17:00 p.m. in a small pool in Ghanna during August 1977. He found no aggregation until 10:00 a.m. but starting from 11:00 a.m., a great mass aggregation of the species was visible on the laterite at the mud/laterite interface (from a muddy to a rocky benthic substrate). The author noted that the small clusters of the ostracods had disappeared by 15:00 p.m. and the main aggregation (or patchiness) was greatly reduced. Eventually, there was no clustering observed by 17:00 p.m. One may interpret Benzie's observation as ostracod species densities might vary depending on the time of a day, but their occurrence (presence/absence) was not limited with the time of sampling, suggesting that ostracods can be collected in any time of a day. Also, his work emphasizes the importance of microhabitat type rather than the macrohabitat type. We did not find significant differences $(P=0.53)$ in the mean numbers of species and abundance collected between 06:30-11:58 a.m. and 12:05-19:52 p.m. This implies that the effect of sampling time in a day may be negligible on the species occurrences. Since there are only a couple of studies on ostracod occurrences and their dayly migration within the same habitat, results need to be further confirmed on daily (and hourly) basis observations in future.

\section{Conclusion}

Overall, these results supported with the previous studies showed that three species ( $H$. incongruens, $I$. inermis, and $I$. bradyi) were most frequently encountered from seven to six different habitats out of 10. In the meantime, we are well aware of that numbers of habitat types where ostracods can be found are not limited with 10 types used herein this study, and we also know that seasonality can be an important factor on ostracods occurrences. Accordingly, underlining these issues (i.e., factors not included here in) that multiple drivers can be effective on species occurrence patterns, we suggest that rare/endemic species can later become cosmopolitan and can be called as "afterwardcosmopolitan species". Furthermore, our findings suggest that most ostracod species reported here exhibited wide distribution among habitats when couple of species (e.g., H. salina) showed certain habitat preferences. Among the variables, water temperature was the most influential factor on species occurrences and their habitat preferences. Finding relatively higher species diversity and abundances in natural habitats than artificial habitats support this view that most ostracods distributed widely prefer better ecological conditions in natural habitats where they cope with optimum conditions. Nevertheless, our results suggest the fact that ostracod occurrences can be related to species-specific characteristics in their $\mathrm{n}$-dimensional niches where species are faced with several other factors.

\section{Acknowledgements}

We thank Dr. Randy Gibson (Aquatic Resources Center, United States Fish and Wildlife Service, San Marcos, Texas, USA) for his comments and help on English revision of the manuscript. Also, Gürkan Özcan and Ozan Yılmaz are thanked for their help during sampling and laboratory works. This study was supported by TÜBITAK (project no: 2130172).

\section{References}

Akdemir, D., Külköylüoğlu, O., Yavuzatmaca, M., \& Sarı, N. (2016). Freshwater ostracods (Crustacea) of Gaziantep (Turkey) and their habitat preferences according to movement ability. Fundamental and Applied Limnology, 187/4, 307-314.

https://dx.doi.org/10.1127/fal/2016/0665.

Belmecheri, S., Namiotko, T., Robert, C., von Grafenstein, U., \& Danielopol, D.L. (2009).

Climate controlled ostracod preservation in Lake Ohrid (Albania, Macedonia). Palaeogeography, Palaeoclimatology, Palaeoecology, 277, 236-245. https://doi.org/10.1016/j.palaeo.2009.04.013

Benson, R.H. (1972). The Bradleya problem, with descriptions of two new psychrospheric ostracode genera, Agrenocythere and Poseidonamicus (Ostracoda: Crustacea). Smithsonian Contributions to Paleobiology, 12, 1-138. https://doi.org/10.5479/si.00810266.12.1

Benzie, J.A.H. (1984). Small scale diurnal migrations by Heterocypris incongruens (Ramdohr, 1808) (Ostracoda: Cyprididae) in a temporary pool, Ghana, West Africa. Journal of Crustacean Biology, 4, 63-65. https://doi.org/10.2307/1547896

Benzie, J.A.H. (1989). The distribution and habitat preference of ostracods (Crustacea Ostracoda) in a coastal sand- 
dune lake, Loch of Strathbeg, north-east Scotland. Freshwater Biology, 22, 309-321. https://doi.org/10.1111/j.1365-2427.1989.tb01104.x Brandão, S.M., \& Yasuhara, M. (2013). Challenging deep-sea cosmopolitanism: taxonomic re-evaluation and biogeography of 'Cythere dasyderma Brady, 1880' (Ostracoda). Journal of Micropalaeontology, 32, 109122. https://doi.org/10.1144/jmpaleo2012-009.

Bronstein, Z.S. (1947). Fresh-water Ostracoda, Fauna of the USSR, Crustaceans. Russian Translation Series, 64. Academy of Sciences of the USSR Publishers, Amerind Publishing Company Moscow, Russia, New Delhi, English translation 1988.

Coleman, B.D. (1981). On random placement and species-area relations. Mathematical Biosciences, 54, 191-215. https://doi.org/10.1016/0025-5564(81)90086-9

Coleman, M.D., Mares, M.D., Willig, M.R., \& Hsieh, Y.H. (1982). Randomness, area, and species richness. Ecology, 63, 1121-1133. https://doi.org/10.2307/1937249

Colwell, R.K., \& Coddington, J.A. (1994). Estimating terrestrial biodiversity through extrapolation. Philosophical Transactions of the Royal Society of London. Series B, Biological Sciences, 345, 101-118. https://doi.org/10.1098/rstb.1994.0091

Fürstenberg, S., Frenzel, P., Peng, P., Henkel, K., \& Wrozyna, C., (2015). Phenotypical variation in Leucocytherella sinensis Huang, 1982 (Ostracoda): a new proxy for palaeosalinity in Tibetan lakes. Hydrobiologia, 751, 5572. https://doi.org/10.1007/s10750-014-2171-3

Ganning, B. (1967). Laboratory experiments in the ecological work on rockpool animals with special notes on the ostracode Heterocypris salinus. Helgol wiss Meeresunters, 15, 27-40.

https://doi.org/10.1007/BF01618607.

Gregory, M.B. (2005). Microhabitat preferences by aquatic invertebrates influence bioassessment metrics in Piedmont streams of Georgia and Alabama. Proceedings of the 2005 Georgia Water Resources Conference, Athens, Georgia.

Griffith, D.M., Veech, J.A., \& Marsh, C.J. (2016). Cooccur: Probabilistic Species Co-Occurrence Analysis in R. Journal of Statistical Software, 69, 1-17. https://doi.org/10.18637/jss.v069.c02

Hart, E.M., \& Gotelli, N.J. (2011). The effects of climate change on density-dependent population dynamics of aquatic invertebrates. Oikos, 120, 1227-1234. https://doi.org/10.1111/j.1600-0706.2011.18707.x

Havel, J.E., Eisenbacher, E.M., \& Black, A.A. (2000). Diversity of crustacean zooplankton in riparian wetlands: colonization and egg banks. Aquatic Ecology, 34, 63-76. https://doi.org/10.1023/A:1009918703131

Hoff, C.C. (1942). The Ostracods of Illinois, their biology and taxonomy. Illinois Biological Monograph, 19, 1-196. https://doi.org/10.5962/bhl.title.50126

Juggins, S. (2003). Software for ecological and palaeoecological data analysis and visualisation, C2 User Guide Version 1.5, University of Newcastle, Newcastle-upon-Tyne, UK.

Karanovic, I. (2012). Recent freshwater ostracods of the world: Crustacea, Ostracoda, Podocopida. Springer-Verlag, Berlin, Heidelberg, Germany. https://doi.org/10.1007/978-3-642-21810-1_3

Kottek et al. 2006. World Map of the Köppen-Geiger climate classification updated. Meteorologische Zeitschrift, 15 (3), 259-263.

https://doi.org/10.1127/0941-2948/2006/0130
Külköylüoğlu, O. (2003). A new report on and the loss of Scottia pseudobrowniana Kempf, 1971 (Ostracoda) from a limnocrene spring in Bolu, Turkey. Crustaceana, 76, 257268. https://doi.org/10.1163/156854003765911667

Külköylüoğlu, O., Meisch, C., \& Rust, W.R. (2003). A new genus (Thermopsis thermophila n. gen.) of Ostracoda (Crustacea) from hot springs of western North America. Hydrobiologia, 499, 113-123. https://doi.org/10.1023/A:1026308514466

Külköylüoğlu, O., Sarı, N., Akdemir, D., Yavuzatmaca, M., \& Altınbağ, C. (2012a). Distribution of Sexual and Asexual Ostracoda (Crustacea) from Different Altitudinal Ranges in the Ordu Region of Turkey: Testing the Rapoport Rule. High Altitude Medicine \& Biology, 13(2), 126-136. http://dx.doi.org/10.1089/ham.2011.1111.

Külköylüoğlu, O., Yavuzatmaca, M., Akdemir, D., \& Sarı, N. (2012b). Distribution and local species diversity of freshwater Ostracoda in relation to habitat in the Kahramanmaraş province of Turkey. International Review of Hydrobiology, 97(4), 247-261. http://dx.doi.org/10.1002/iroh.201111490.

Külköylüoğlu, O., Sarı, N., \& Akdemir, D. (2012c). Distribution and ecological requirements of ostracods (Crustacea) at high altitudinal ranges in Northeastern Van (Turkey). Annales de Limnologie - International Journal of Limnology, 48, 39-51. http://dx.doi.org/10.1051/limn/2011060.

Külköylüoğlu, O., Akdemir, D., \& Yüce, R. (2012d). Distribution, ecological tolerance and optimum levels of freshwater Ostracoda (Crustacea) from Diyarbakır, Turkey. Limnology, 13, 73-80. https://doi.org/10.1007/s10201011-0357-1

Külköylüoğlu, O., Yavuzatmaca, M., Sarı, N., \& Akdemir, D. (2016). Elevational distribution and species diversity of freshwater Ostracoda (Crustacea) in Çankırı region (Turkey). Journal of Freshwater Ecology, 31, 219-230. https://doi.org/10.1080/02705060.2015.1050467.

Külköylüoğlu, O., Yavuzatmaca, M., Tanyeri, M., \& Yılmaz, O. (2017a). Ostracoda (Crustacea) species composition and environmental correlates in different aquatic habitats of the Zonguldak and Bartın regions (Turkey). Turkish Journal of Zoology, 41, 686-695. https://doi.org/10.3906/zoo-1512-36

Külköylüoğlu, O., Yılmaz, S., \& Yavuzatmaca, M. (2017b). Comparison of Ostracoda (Crustacea) species distribution, diversity and ecological characteristics among habitat types. Fundamental and Applied Limnology, 190(1), 63-86. https://doi.org/10.1127/fal/2017/0872

Külköylüoğlu, O., \& Vinyard, G.L. (2000). Distribution and ecology of freshwater Ostracoda (Crustacea) collected from springs of Nevada, Idaho and Oregon: A preliminary study. West North American Naturalist, 60, 291-303. https://scholarsarchive.byu.edu/wnan/vol60/iss3/6

Laprida, C., Díaz, A., \& Ratto, N. (2006). Ostracods (Crustacea) from thermal waters, Southern Altiplano, Argentina. Micropaleontology, 52, 177-188. https://doi.org/10.2113/gsmicropal.52.2.177

Lopez, L.C.S., Rodrigues, P.J.F.P., \& Rios, R.I. (1999). Frogs and snakes as phoretic dispersal agents of Bromeliad ostracods (Limnocytheridae: Elpidium) and annelids (Naididae: Dero). Biotropica, 31(4), 705-708. https://doi.org/10.1111/j.1744-7429.1999.tb00421.x

McLay, C.L. (1978a). Comparative observations on the ecology of four species of ostracods living in a temporary 
freshwater puddle. Canadian Journal of Zoology, 56, 663-675. https://doi.org/10.1139/z78-094

McLay CL (1978b). The population biology of Cyprinotus carolinensis and Herpetocypris reptans (Crustacea, Ostracoda). Canadian Journal of Zoology, 56, 1170-1179. https://doi.org/10.1139/z78-161

Meisch, C. (2000). Freshwater Ostracoda of western and central Europe. Heidelberg, Spektrum Akademischer Verlag, Süßwasserfauna von Mitteleuropa.

Mischke, S., Herzschuh, U., Massmann, G., \& Zhang, C. (2007). An ostracod-conductivity transfer function for Tibetan lakes. Journal of Paleolimnology, 38, 509-524. https://doi.org/10.1007/s10933-006-9087-5

Moreno, E., Pérez-Martínez, C., Conde-Porcuna, J.M. (2016). Dispersal of zooplankton dormant propagules by wind and rain in two aquatic systems. Limnetica, 35, 323-336. https://doi.org/10.23818/limn.35.26

Panuccio, M., Agostini, N., Nelli, L., Andreou, G., \& Xirouchachis, S. (2019). Factors shaping distribution and abundance of raptors wintering in two large Mediterranean islands. Community Ecology, 20, 93-103. https://doi.org/10.1556/168.2019.20.1.10

Proctor, V.W., Malone, C.R., \& De Vlaming, V.L. (1967). Dispersal of aquatic organisms: viability of disseminules recovered from the intestinal tract of captive killdeer. Ecology, 48, 672-676. https://doi.org/10.2307/1936517

Rahel, F.J., \& Olden, J.D. (2008). Assessing the effects of climate change on aquatic invasive species. Conservation Biology, 22, 521-533. https://doi.org/10.1111/j.1523-1739.2008.00950.x

Scharf, B., \& Viehberg, F.A. (2014). Living Ostracoda (Crustacea) from the town Moat of Bremen, Germany. Crustaceana, 87, 1124-1135. https://doi.org/10.1163/15685403-00003345

Seaby, R.M., \& Henderson, P.A. (2006). Species Diversity and Richness. Version 4. Pisces Conservation Ltd. Lymington, England.

Ter Braak, C.J.F. (1995). Ordination. In R.H.G. Jongman, C.J.F. ter Braak \& O.F.R. Van Tongeren (Eds.), Data Analysis in community and landscape ecology (pp. 91-173). Cambridge University Press, Cambridge. https://doi.org/10.1017/СВO9780511525575

Turkish State Meteorological Service (TSMS). 2020. https://mgm.gov.tr/veridegerlendirme/il-ve-ilceleristatistik.

Uçak, S., Külköylüoğlu, O., Akdemir, D., \& Başak, E. (2014). Distribution, Diversity and Ecological Characteristics of
Freshwater Ostracoda (Crustacea) in Shallow Aquatic Bodies of the Ankara Region, Turkey. Wetlands, 34, 309324.

http://dx.doi.org/10.1007/s13157-013-0499-5.

Valls, L., Castillo-Escrivà, A., Barrera, L., Gómez, E., Gil-Delgado, J.A., Mesquita-Joanes, F., \& Armengol, X. (2017). Differential endozoochory of aquatic invertebrates by two duck species in shallow lakes. Acta Oecologica, 80, 39-46. https://doi.org/10.1016/j.actao.2017.03.003

Veech, J.A. (2013). A probabilistic model for analysing species co-occurrence. Global Ecology and Biogeography 22: 252 $-260$.

https://doi.org/10.1111/j.1466-8238.2012.00789.x

Veech, J.A. (2014). The pairwise approach to analysing species cooccurrence. Journal of Biogeography, 41, 1029-1035. https://doi.org/10.1111/jbi.12318

Vinyard, G. (1979). An ostracod (Cypriodopsis vidua) can reduce predation from fish by resisting digestion. American Midland Naturalist, 102, 188-190. https://doi.org/10.2307/2425084

Wanschoenwinkel, B., Waterkeyn, A., Vandecaetsbeek, T., Pineau, O., Grillas, P., \& Brendonck, L. (2008). Dispersal of freshwater invertebrates by large terrestrial mammals: a case study with wild boar (Sus scrofa) in Mediterranean wetlands. Freshwater Biology, 53, 22642273.

https://doi.org/10.1111/j.1365-2427.2008.02071.x

Wise, C.D. (1961). Taxonomy and Ecology of fresh-water ostracods of south-central Texas. [Unpublished doctoral dissertation]. The University of New Mexico.

Yavuzatmaca, M., Külköylüoğlu, O., \& Yılmaz, O. (2015). Distributional patterns of non-marine Ostracoda (Crustacea) in Adıyaman Province (Turkey). Annales de Limnologie -International Journal of Limnology, 51, 101113. http://dx.doi.org/10.1051/limn/2015005.

Yavuzatmaca, M., Külköylüoğlu, O., \& Yılmaz, O. (2017). Estimating distributional patterns of non-marine Ostracoda (Crustacea) and habitat suitability in the Burdur province (Turkey). Limnologica, 62, 19-33. http://dx.doi.org/10.1016/j.limno.2016.09.006.

Yavuzatmaca, M., Külköylüoğlu, O., Akdemir, D., \& Çelen, E. (2018). On the relationship between the occurrence of ostracod species and elevation in Sakarya Province, Turkey. Acta zoologica Academiae Scientiarum Hungaricae, 64, 329-354.

https://doi.org/10.17109/AZH.64.4.329.2018. 